\title{
ODD PRIMARY STEENROD OPERATIONS IN FIRST-QUADRANT SPECTRAL SEQUENCES
}

BY

\author{
JOHN SAWKA
}

\begin{abstract}
This paper defines two kinds of Steenrod operations in the spectral sequence of a bisimplical mod $p$ coalgebra and shows them to be a complete list of all such possible Steenrod operations. These operations are compatible with the differentials and with Steenrod operations on the total complex. A general rule is given for computing the operations on $E_{2}$. A generalization of the Kudo transgression theorem is also proved, placing it in a larger and more natural setting.
\end{abstract}

1. Introduction. In 1955, Massey [9] asked if it were possible to introduce Steenrod squares and $p$ th powers into the spectral sequence of a fibre space. Since that time several people have contributed to the solution of this problem, notably Vazquez [22], Araki [1], Kristensen [5], Kuo [7], Rector [15], Smith [18], Singer [16], Mori ([12] and [13]), and Dwyer [4].

This paper will define mod $p$ Steenrod operations on a class of spectral sequences; its sequel will define higher divided powers in certain spectral sequences. These operations are shown to be a complete list of all Steenrod-type operations on spectral sequences. This extends to the odd prime case the work of Singer [16] on Steenrod squares in first-quadrant spectral sequences and the work of Dwyer [4] on higher divided squares in second quadrant spectral sequences. Mori ([12] and [13]) has also obtained most of our results for the odd prime case of the Eilenberg-Moore spectral sequence through different methods.

In the course of these papers the ambiguity regarding indeterminacy in the definition of the operations in [16], [12], and [13] is resolved, and the question asked by Singer [16] regarding the existence of horizontal operations in the spectral sequence of a mixed simplicial object is answered. A generalization of the Kudo transgression theorem [6] is also proved, placing the usual Kudo transgression theorem in a larger and more natural setting. The principal applications of these results are to the Eilenberg-Moore and the Serre spectral sequences as well as to the change-of-rings spectral sequence as outlined by Singer in [17].

Let $\Delta$ be the category of finite ordered sets and nondecreasing maps [10,p. 4] and let $\Delta^{\text {op }}$ be the opposite category. A bisimplicial coalgebra is a covariant functor from

Received by the editors September 10, 1980 and, in revised form, August 4, 1981.

1980 Mathematics Subject Classification. Primary 55T05, 55S10; Secondary 55T10, 55T20, 55R20, 55U10, 18G40.

Key words and phrases. Steenrod operations, spectral sequence, cohomology of fibre spaces, cohomology of Hopf algebras, Eilenberg-Moore spectral sequence, Kudo transgression theorem. 
$\Delta^{\mathrm{op}} \times \Delta^{\mathrm{op}}$ to the category of commutative $Z_{p}$ coalgebras. Throughout this paper $p$ is an odd prime. A mixed simplicial coalgebra is a covariant functor from $\Delta^{\mathrm{op}} \times \Delta$ to the category of commutative $Z_{p}$ coalgebras.

The vector space dual of a bisimplicial coalgebra can be regarded as a double cochain complex and filtered in the usual way to give a first-quadrant spectral sequence. The main result of this paper is the construction in this spectral sequence of vertical Steenrod operations

$$
\begin{array}{rlll}
\mathcal{P}^{k}: E_{r}^{s, t} & \rightarrow & E_{r}^{s, t+2 k(p-1)} & (0 \leqslant 2 k \leqslant t), \\
\beta \mathcal{P}^{k}: E_{r}^{s, t} & \rightarrow & E_{r}^{s, t+2 k(p-1)+1} & (0 \leqslant 2 k<t),
\end{array}
$$

and horizontal Steenrod operations

$$
\begin{aligned}
& \mathscr{P}^{k}: E_{r}^{s, t} \rightarrow E_{r}^{s+(2 k-t)(p-1), p t} \\
& \beta \mathcal{P}^{k}: E_{r}^{s, t} \rightarrow E_{r}^{s+(2 k-t)(p-1)+1, p t} \\
& (t \leqslant 2 k \leqslant t+r-2)
\end{aligned}
$$

of certain indeterminacy (detailed later). Note that the $\beta^{\mathcal{P}^{\mathbf{k}}}$ is a single operation which is not a priori related to any Bockstein operations. Proposition 2.5 shows that these operations commute with the differentials of the spectral sequence. In particular, they are defined on $E_{\infty}$, where they are compatible with the action of Steenrod operations on the cohomology of the total complex (Proposition 2.4).

A mixed simplicial coalgebra similarly gives rise to a second-quadrant spectral sequence. The main result of the sequel to this paper is the construction in this spectral sequence of vertical Steenrod operations

$$
\begin{array}{rlll}
\mathcal{P}^{k}: E_{r}^{-s, t} & \rightarrow & E_{r}^{-s, t+2 k(p-1)} & (0 \leqslant 2 k \leqslant t), \\
\beta^{\mathcal{P}^{k}}: E_{r}^{-s, t} & \rightarrow & E_{r}^{-s, t+2 k(p-1)+1} & (0 \leqslant 2 k<t),
\end{array}
$$

and horizontal higher divided power operations

$$
\begin{array}{rll}
\delta_{k}: E_{r}^{-s, t} & \rightarrow E_{r}^{-s-2 k(p-1)-1, p t} & (t \text { even }), \\
\delta_{k}: E_{r}^{-s, t} & \rightarrow E_{r}^{-s-(2 k-1)(p-1)-1, p t} & (t \text { odd }), \\
\beta \delta_{k}: E_{r}^{-s, t} & \rightarrow E_{r}^{-s-2 k(p-1), p t} & (t \text { even }), \\
\beta \delta_{k}: E_{r}^{-s, t} & \rightarrow E_{r}^{-s-(2 k-1)(p-1), p t} & (t \text { odd })
\end{array}
$$

of certain indeterminacy to be detailed later. These higher divided powers provide the classes necessary to enable the differentials to kill superfluous Steenrod operations, thus ensuring that at the $E_{\infty}$ stage, the action of the Steenrod algebra is unstable with respect to total degree.

The techniques used in both papers are those of Dwyer ([3] and [4]) and Singer [16]. It is a pleasure to acknowledge my debt to them, particularly to W. G. Dwyer who provided invaluable advice during the course of this work. I would also like to thank the referee for his helpful comments.

2. Definitions and results. For an algebra or coalgebra $\Lambda$ over $Z_{p}$, we denote the comultiplication by $\psi: \Lambda \rightarrow \Lambda \otimes \Lambda$ and the multiplication by $\mu: \Lambda \otimes \Lambda \rightarrow \Lambda$. We write $\varepsilon: \Lambda \rightarrow Z_{p}$ and $\eta: Z_{p} \rightarrow \Lambda$ for the counits and units, respectively. If $\Lambda$ is a Hopf 
algebra, then a left $\Lambda$-coalgebra is a coalgebra $M$ that is also a left $\Lambda$-module such that $\psi: M \rightarrow M \otimes M$ and $\varepsilon: M \rightarrow Z_{p}$ are $\Lambda$-morphisms. Here $\Lambda$ acts diagonally on $M \otimes M$, with all tensor products taken over $Z_{p}$.

The constituents of a bisimplicial object $X$ are written $X_{s, t}$, and $d_{i}^{v}, s_{i}^{v}$ designate the vertical face and degeneracy operators, while $d_{i}^{h}, s_{i}^{h}$ denote the horizontal operators. An augmentation for a bisimplicial object $X$ is a simplicial object $R$ and a morphism of simplicial objects $\lambda: X_{0, *} \rightarrow R_{*}$ such that $\lambda d_{1}^{h}=\lambda d_{0}^{h}: X_{1, t} \rightarrow R_{t}$ for all $t \geqslant 0$.

If $\Lambda$ is a Hopf algebra and $R$ is a simplicial $\Lambda$-module, then there is an associated $\Lambda$-chain complex $C R$ defined by $(C R)_{n}=R_{n}$ and $d=\sum_{i=0}^{n}(-1)^{i} d_{i}$. Similarly, for a bisimplicial $\Lambda$-module $X$, there is an associated double $\Lambda$-chain complex $C X$ defined by $(C X)_{s, t}=X_{s, t}$ with $d^{v}=\Sigma_{i=0}^{t}(-1)^{i} d_{i}^{v}$ and $d^{h}=\Sigma_{j=0}^{s}(-1)^{j} d_{j}^{h}$. We can also regard $C X$ as a singly-indexed $\Lambda$-chain complex with increasing filtration:

$$
F_{k}(C X)_{n}=\sum_{\substack{i+j=n \\ i \leqslant k}} C X_{i, j}
$$

Here the total differential on an element $x$ of bidegree $(s, t)$ is given by $d x=d^{h} x+$ $(-1)^{s} d^{v} x$.

If $X$ is a bisimplicial $\Lambda$-module and $N$ is a $\Lambda$-module, we write $\operatorname{Hom}_{\Lambda}(C X, N)$ for the $Z_{p}$-cochain complex with decreasing filtration given by

$$
F^{k} \operatorname{Hom}_{\Lambda}^{n}(C X, N)=\left\{f:(C X)_{n} \rightarrow N \mid f\left(F_{k-1} C X\right)=0\right\} .
$$

We write $\left(E_{r} \operatorname{Hom}_{\Lambda}(C X, N), d_{r}\right)$, or just $\left(E_{r}, d_{r}\right)$ for the spectral sequence of this filtered cochain complex. This is called the spectral sequence of the bisimplicial $\Lambda$-coalgebra and is just a special case of the spectral sequence of a bisimplicial $\Lambda$-module. If $X$ has an augmentation $\lambda: X \rightarrow R$, then $\lambda$ defines in an obvious way a $Z_{p}$-homomorphism

$$
\lambda^{*}: H^{*} \operatorname{Hom}_{\Lambda}(C R, N) \rightarrow H^{*} \operatorname{Hom}_{\Lambda}(C X, N) .
$$

When $\lambda^{*}$ is an isomorphism, then $H^{*} \operatorname{Hom}_{\Lambda}(C R, N)$ is filtered and is the target of the spectral sequence $\left(E_{r}, d_{r}\right)$. In this case, write

$$
\rho: F^{s} H^{s+t} \operatorname{Hom}_{\Lambda}(C R, N) \rightarrow E_{\infty}^{s, t}
$$

for the usual projection.

Finally, for $2 \leqslant r \leqslant n$, let $B_{n}^{s, t}$ denote the subgroup of $E_{r}^{s, t}$ containing those elements which survive to $E_{n}^{s, t}$ and have zero residue class in $E_{n}^{s, t}$. An operation $f$ : $E_{r}^{s, t} \rightarrow E_{r}^{u, v}$ of indeterminacy $n$ is a map

$$
E_{r}^{s, t} \rightarrow E_{r}^{u, v} / B_{n}^{u, v} \text {. }
$$

We can now state our results concerning the (first-quadrant) spectral sequence of a bisimplicial $\Lambda$-coalgebra.

Suppose that $\Lambda$ is a Hopf algebra, $R$ is a simplicial $\Lambda$-coalgebra, $X$ is a bisimplicial $\Lambda$-coalgebra, and $N$ is a commutative $\Lambda$-algebra. In $\$ 4$, products and Steenrod operations in $H^{*} \operatorname{Hom}_{\Lambda}(C R, N)$ are defined. In $\$ 5$, products and Steenrod operations are defined on $H^{*} \operatorname{Hom}_{\Lambda}(C X, N)$. 
Proposition 2.1. If $\lambda: X \rightarrow R$ is an augmentation, then $\lambda^{*}: H^{*} \operatorname{Hom}_{\Lambda}(C R, N) \rightarrow$ $H^{*} \operatorname{Hom}_{\Lambda}(C X, N)$ preserves products and Steenrod operations.

In $\S 5$, we also define products in the spectral sequence $\left(E_{r} \operatorname{Hom}_{\Lambda}(C X, N), d_{r}\right)$ :

$$
E_{r}^{s, t} \otimes E_{r}^{s^{\prime}, t^{\prime}} \rightarrow E_{r}^{s+s^{\prime}, t+t^{\prime}},
$$

as well as two kinds of Steenrod operations. We define vertical Steenrod operations

$$
\begin{aligned}
\mathcal{P}^{k}: E_{r}^{s, t} \rightarrow E_{r}^{s, t+2 k(p-1)} & (0 \leqslant 2 k \leqslant t), \\
\beta^{\mathcal{P}^{k}}: E_{r}^{s, t} \rightarrow E_{r}^{s, t+2 k(p-1)+1} & (0 \leqslant 2 k<t),
\end{aligned}
$$

with no indeterminacy. We define horizontal Steenrod operations

$$
\begin{aligned}
\mathcal{P}^{k}: E_{r}^{s, t} & \rightarrow E_{r}^{s+(2 k-t)(p-1), p t} \\
\beta \mathcal{P}^{k}: E_{r}^{s, t} & \rightarrow E_{r}^{s+(2 k-t)(p-1)+1, p t} \quad(t \leqslant 2 k),
\end{aligned}
$$

which are of indeterminacy $r-1+(2 k-t)(p-1)$ and $r+(2 k-t)(p-1)$, respectively, for $t \leqslant 2 k<t+r-2$, and of indeterminacy $p(r-2)+1$ for $t+r-$ $2 \leqslant 2 k$. Note that at the $E_{2}$ level there is no indeterminacy.

Proposition 2.2. Products and Steenrod operations on $E_{2}$ determine products and Steenrod operations on $E_{r}$ (modulo the appropriate indeterminacy) for all $r \geqslant 2$; that is, if $u \in E_{2}$ survives to $E_{r}$ and represents $[u] \in E_{r}$, then $\mathcal{P}^{k} u$ and $\beta^{\mathcal{P}^{k}} u$ survive to $E_{r}$, $\mathscr{P}^{k}[u]=\left[\mathscr{P}^{k} u\right]$, and $\beta^{\mathscr{P}^{k}}[u]=\left[\beta^{\mathcal{P}^{k}} u\right]$ modulo the appropriate indeterminacy.

Proposition 2.3. The spectral sequence $\left(E_{r}, d_{r}\right)$ is a differential algebra under the product pairing.

It follows that there are products and Steenrod operations on $E_{\infty}$. The following proposition relates them to those of the target of the spectral sequence.

Proposition 2.4. Suppose that $X$ is a bisimplicial $\Lambda$-coalgebra with augmentation $\lambda$ : $X \rightarrow R, N$ is a commutative $\Lambda$-algebra, and $\lambda^{*}$ is an isomorphism. Suppose that $u \in F^{s} H^{s+t} \operatorname{Hom}_{\Lambda}(C R, N)$ and $v \in F^{m} H^{m+n} \operatorname{Hom}_{\Lambda}(C R, N)$. Then $u v \in$ $F^{s+m} H^{*} \operatorname{Hom}_{\Lambda}(C R, N)$ and $\rho(u v)=\rho(u) \rho(v)$. In addition we have the following:

Case 1. If $0 \leqslant 2 k \leqslant t$, then $\rho^{k} u \in F^{s} H^{*} \operatorname{Hom}_{\Lambda}(C R, N)$ and $\rho^{\mathcal{P}^{k}} u=\mathcal{P}^{k} \rho u$. If $0 \leqslant 2 k<t$, then $\beta^{\mathcal{P}^{k}} u \in F^{s} H^{*} \operatorname{Hom}_{\Lambda}(C R, N)$ and $\rho \beta \mathcal{P}^{k} u=\beta \mathcal{P}^{k} \rho u$.

Case 2. If $t \leqslant 2 k$, then

$$
\begin{gathered}
\mathcal{P}^{k} u \in F^{s+(2 k-t)(p-1)} H^{*} \operatorname{Hom}_{\Lambda}(C R, N), \\
\beta^{\rho^{k}} u \in F^{s+(2 k-t)(p-1)+1} H^{*} \operatorname{Hom}_{\Lambda}(C R, N)
\end{gathered}
$$

and

$$
\rho^{\rho^{k}} u=\mathscr{P}^{k} \rho u, \quad \rho \beta \mathscr{P}^{k} u=\beta \mathscr{\rho}^{k} \rho u .
$$

The Steenrod operations we define commute with the differentials in the spectral sequence in the manner described in the next proposition. In order to simplify the notation, no distinction is made between an element in $E_{r}$ that survives to $E_{s}$ and its residue class in $E_{s}$. 
Proposition 2.5. Suppose that $u \in E_{r}^{s, t}(r \geqslant 2)$.

Case 1. If $2 k \leqslant t-r+1$, then

$$
d_{r} \rho^{k} u=\rho^{k} d_{r} u
$$

and if $2 k<t-r+1$, then

$$
d_{r} \beta \mathscr{P}^{k} u=-\beta \mathscr{P}^{k} d_{r} u
$$

Case 2. If $t-r+1 \leqslant 2 k \leqslant t$, then $\mathcal{P}^{k} u$ survives to $E_{q}^{s, t+2 k(p-1)}$ where $q=r+$ $(2 k-t+r-1)(p-1), \rho^{k} d_{r} u$ survives to $E_{q}^{s+q, p(t-r+1)}$, and

$$
d_{q} \mathcal{P}^{k} u=\mathscr{P}^{k} d_{r} u
$$

If $t-r+1 \leqslant 2 k<t$, then $\beta^{\mathcal{P}^{k}} u$ survives to $E_{q+1}^{s, t+2 k(p-1)+1}, \beta^{\mathcal{P}^{k}} d_{r} u$ survives to $E_{q+1}^{s+q+1, p(t-r+1)}$, and

$$
d_{q+1} \beta \mathscr{P}^{k} u=-\beta \mathscr{P}^{k} d_{r} u
$$

Case 3. If $t \leqslant 2 k$, then $\mathcal{P}^{k} u$ survives to $E_{p(r-1)+1}^{s+(2 k-t)(p-1), p t}, \mathscr{P}^{k} d_{r} u$ survives to $E_{p(r-1)+1}^{s+r+(2 k-t+r-1)(p-1), p(t-r+1)}$, and

$$
d_{p(r-1)+1} \rho^{k} u=\mathcal{P}^{k} d_{r} u .
$$

In addition, $\beta \mathscr{P}^{k} u$ survives to $E_{p(r-1)+1}^{s+(2 k-t)(p-1)+1, p t}, \beta \mathscr{P}^{k} d_{r} u$ survives to $E_{p(r-1)+1}^{s+r+(2 k-t+r-1)(p-1)+1, p(t-r+1)}$, and

$$
d_{p(r-1)+1} \beta \mathscr{P}^{k} u=-\beta \mathscr{P}^{k} d_{r} u
$$

Case 4. If $s+t$ is even, $s+t=2 k$, and $d_{r} v=u$, then

$$
d_{r} v u^{(p-1)}=\mathscr{\rho}^{k} u \text {. }
$$

If $s+t$ is odd, $s+t-1=2 k$, and $d_{r} v=u$, then $\beta^{\rho^{k}} u$ and $v^{(p-1)} u$ both survive to $E_{(p-1)(r-1)+1}$ and

$$
d_{(p-1)(r-1)+1} v^{(p-1)} u=\beta \rho^{k} u .
$$

In order to better understand Proposition 2.5, consider the following diagram. Each of the numbers, $1,2,3$, and 4 , indicates the region determined by the hypothesis of the corresponding case of the proposition.

These results are analogous to those of Singer [16] for the mod 2 case. Mori ([12] and [13]) obtains the first three cases of our Proposition 2.5 for the $\bmod p$ Eilenberg-Moore spectral sequence. Neither Singer nor Mori, however, explicitly mentions the indeterminacy inherent in the operations.

We can summarize Proposition 2.5 informally by saying that $\mathcal{P}^{k} u$ and $\mathcal{P}^{k} d_{r} u$ (or $\beta^{\mathcal{P}^{k}} u$ and $\beta \mathcal{P}^{k} d_{r} u$ ) survive long enough for $\mathscr{P}^{k} u$ to "kill" $\mathscr{P}^{k} d_{r} u$ (or for $\beta^{\mathcal{P}^{k}} u$ to "kill" $\beta \rho^{k} d_{r} u$ ). In order to see how long these classes need to survive, make a sketch and observe the value of $q$ required for $d_{q}$ to take $\mathscr{P}^{k} u$ to $\mathcal{P}^{k} d_{r} u$. The several cases arise because the operations switch from being vertical to horizontal at different places for $u$ and $d_{r} u$. 


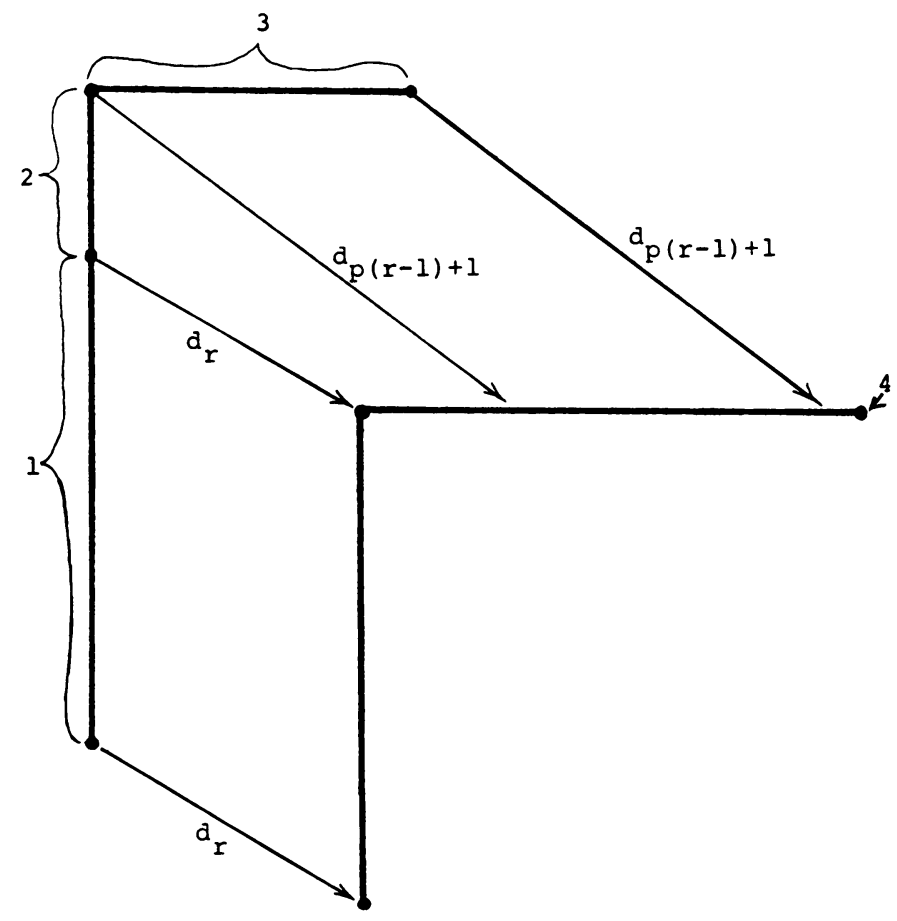

Case 4 generalizes the Kudo transgression theorem [6] and places it in a natural setting. The class $d_{r} v$ is of one higher total degree than $v$, so there is one more operation defined on it than on $v$. Case 4 provides a class to hit this additional operation.

3. Simplicial Eilenberg-Zilber maps. For $R$ and $S$ simplicial $\Lambda$-modules, we write $R \otimes S$ for the dimensionwise tensor product: $(R \otimes S)_{n}=R_{n} \otimes S_{n}$ with diagonal $\Lambda$-action and tensor product face and degeneracy operators. We also write $C R \hat{\otimes} C S$ for the (graded) tensor product of $\Lambda$-chain complexes: $(C R \hat{\otimes} C S)_{n}=\sum_{i+j=n} R_{i} \otimes S_{j}$ with Leibnitz differential

$$
d(a \otimes b)=d(a) \otimes b+(-1)^{|a|} a \otimes d(b) .
$$

These definitions extend in the obvious way to $p$ factors.

Suppose that $R(1), R(2), \ldots, R(p)$ are simplicial $\Lambda$-modules. Then let

$$
T: C(R(1) \otimes R(2) \otimes \cdots \otimes R(p)) \rightarrow C(R(p) \otimes R(1) \otimes \cdots \otimes R(p-1))
$$

or

$$
T: C R(1) \otimes C R(2) \otimes \cdots \otimes C R(p) \rightarrow C R(p) \otimes C R(1) \otimes \cdots \otimes C R(p-1)
$$

denote the signed cyclic permutation of factors:

$$
T\left(a_{1} \otimes a_{2} \otimes \cdots \otimes a_{p}\right)=(-1)^{\left|a_{p}\right|\left(\left|a_{1}\right|+\left|a_{2}\right|+\cdots+\left|a_{p-1}\right|\right)}\left(a_{p} \otimes a_{1} \otimes a_{2} \otimes \cdots \otimes a_{p-1}\right) .
$$

LEMMA 3.1. For $R(1), R(2), \ldots, R(p)$ simplicial $\Lambda$-modules, there exist natural maps

$$
\begin{aligned}
D_{k} & =D_{k}(R(1), R(2), \ldots, R(p)): C(R(1) \otimes R(2) \otimes \cdots \otimes R(p)) \\
& \rightarrow C R(1) \hat{\otimes} C R(2) \hat{\otimes} \ldots \hat{\otimes} C R(p) \quad(k \geqslant 0)
\end{aligned}
$$


homogeneous of degree $k$ such that

(i) $D_{0}$ is a chain homotopy equivalence which induces the identity map $C(R(1) \otimes R(2) \otimes \cdots \otimes R(p))_{0} \rightarrow(C R(1) \hat{\otimes} C R(2) \hat{\otimes} \cdots \hat{\otimes} C R(p))_{0}$,

(ii) $d D_{k}+D_{k} d=D_{k-1}-T D_{k-1} T^{-1}$ for $k$ odd, and

(iii) $d D_{k}-D_{k} d=D_{k-1}+T D_{k-1} T^{-1}+\cdots+T^{p-1} D_{k-1} T^{-p+1}$ for $k$ even.

The collection $\left\{D_{k}\right\}$ is called a simplicial Eilenberg-Zilber map in the sense of [16] and is easily constructed via acyclic models as in [21, p. 441] or [2].

A simplicial Eilenberg-Zilber map $\left\{D_{k}\right\}$ is called special if for all $R(1), R(2), \ldots, R(p)$, and for all integers $n, k$, we have the following two conditions.

(1) The image of

$$
D_{k}: C(R(1) \otimes R(2) \otimes \cdots \otimes R(p))_{n} \rightarrow(C R(1) \hat{\otimes} C R(2) \hat{\otimes} \cdots \hat{\otimes} C R(p))_{n+k}
$$

is contained in the subspace

$$
\sum_{i_{1}, i_{2}, \ldots, i_{p} \leqslant n} C R(1)_{i_{1}} \otimes C R(2)_{i_{2}} \otimes \cdots \otimes C R(p)_{i_{p}},
$$

(In particular, $D_{k}\left(C(R(1) \otimes \cdots \otimes R(p))_{n}\right)=0$ if $k>(p-1) n$.)

(2) The map

$$
D_{(p-1) n}: C(R(1) \otimes R(2) \otimes \cdots \otimes R(p))_{n} \rightarrow(C R(1) \hat{\otimes} C R(2) \hat{\otimes} \cdots \hat{\otimes} C R(p))_{p n}
$$

(for $n>0$ ) is given by

$$
D_{(p-1) n}\left(a_{1} \otimes a_{2} \otimes \cdots \otimes a_{p}\right)=(m !)^{n}(-1)^{m\left(n^{2}+n\right) / 2} a_{1} \otimes a_{2} \otimes \cdots \otimes a_{p}
$$

for all $a_{i} \in R(i)$ where $m=(p-1) / 2$.

By using normalized complexes in the proof of Lemma 3.1, as in [19, p. 274], we can always be sure that condition (1) is satisfied. That condition (2) can always be satisfied is shown as Theorem 5.4 in [20]. We can always, therefore, insist that our Eilenberg-Zilber map is special.

If $N$ is a $\Lambda$-module, we also consider the dual morphism

$$
D_{k}^{*}: \operatorname{Hom}_{\Lambda}(C R(1) \hat{\otimes} \cdots \hat{\otimes} C R(p), N) \rightarrow \operatorname{Hom}_{\Lambda}(C(R(1) \otimes \cdots \otimes R(p)), N),
$$

which satisfies formulas dual to those of Lemma 3.1.

4. Products and Steenrod operations for simplicial coalgebras. Suppose that $N$ is a commutative $\Lambda$-algebra with product $\mu: N \otimes N \rightarrow N$ and that $R$ is a simplicial coalgebra with coproduct $\psi: R \rightarrow R \otimes R$. Let

$$
\chi: \operatorname{Hom}_{\Lambda}(C R, N) \hat{\otimes} \operatorname{Hom}_{\Lambda}(C R, N) \rightarrow \operatorname{Hom}_{\Lambda}(C R \hat{\otimes} C R, N)
$$

be the natural pairing that takes $x: C R \rightarrow N, y: C R \rightarrow N$ to $C R \hat{\otimes} C R \stackrel{x \otimes y}{\rightarrow} N \otimes$ $N \stackrel{\mu}{\rightarrow} N$. Also write $\chi$ for the obvious analog for $p$ factors. We can now define cochain operations

$$
\begin{aligned}
& \mu: \operatorname{Hom}_{\Lambda}^{s}(C R, N) \otimes \operatorname{Hom}_{\Lambda}^{t}(C R, N) \rightarrow \operatorname{Hom}_{\Lambda}^{s+t}(C R, N), \\
& P^{k}: \operatorname{Hom}_{\Lambda}^{n}(C R, N) \rightarrow \operatorname{Hom}_{\Lambda}^{n+2 k(p-1)}(C R, N), \text { and } \\
& \beta P^{k}: \operatorname{Hom}_{\Lambda}^{n}(C R, N) \rightarrow \operatorname{Hom}_{\Lambda}^{n+2 k(p-1)+1}(C R, N) .
\end{aligned}
$$


First, define $\mu$ by $\mu(x \otimes y)=\psi^{*} D_{0}^{*} \chi(x \otimes y)$ where $D_{0}$ is the natural analog for two factors of the similar map defined in $\S 3$ for $p$ factors.

In order to define $P^{k}$ and $\beta P^{k}$ we need first to define certain cochains and we follow May [11, pp. 166-167]. Let $x \in \operatorname{Hom}_{\Lambda}^{n}(C R, N)$ and $d x=y$ $\in \operatorname{Hom}_{\Lambda}^{n+1}(C R, N)$. Let $C$ be the free $Z_{p}$-cochain complex with generators $\tilde{x}$ in dimension $n$ and $\tilde{y}$ in dimension $n+1$ with $d \tilde{x}=\tilde{y}$ and all other differentials zero. Define a contraction $s: C \rightarrow C$ of degree minus one by $s(\tilde{x})=0, s(\tilde{y})=\tilde{x}$. Then $d s+s d=1$. Extend $s$ to $S: C^{p} \rightarrow C^{p}$ by $S=1^{p-1} \otimes s$. It follows that $S$ is given explictly by $S(a \tilde{x})=0, S(a \tilde{y})=(-1)^{|a|} a \tilde{x}$ for $a \in C^{p-1}$. Also $d S+S d=1$. Next, define $\tilde{t}_{i}$ for $0 \leqslant i \leqslant p$ inductively by

$$
\begin{gathered}
\tilde{t}_{0}=\tilde{y}^{p} ; \quad \tilde{t}_{1}=\tilde{y}^{p-1} \tilde{x}, \\
\tilde{t}_{2 j}=S\left(\left(1-T^{-1}\right) \tilde{t}_{2 j-1}\right) ; \quad \tilde{t}_{2 j+1}=S\left(N \tilde{t}_{2 j}\right)
\end{gathered}
$$

where $N=1+T+T^{2}+\cdots+T^{p-1}$. Using the fact that $d S+S d=1$, we find

(i) $d \tilde{t}_{1}=\tilde{t_{0}}$,

(ii) $d \tilde{t}_{2 j}=\left(1-T^{-1}\right) \tilde{t}_{2 j-1}$, and

(iii) $d \tilde{t}_{2 j+1}=N \tilde{t}_{2 j}$.

By induction we can explicitly calculate $\tilde{t}_{i}$. Here we merely note that $\tilde{t}_{p}=$ $(-1)^{m(n+1)} m ! \tilde{x}^{p}$, where here and in the following $m=(p-1) / 2$. Now let $t_{i}$ be the image in $\left(\operatorname{Hom}_{\Lambda}(C R, N)\right)^{p}$ of $\tilde{t}_{i}$ under $\alpha^{p}$ where $\alpha: C \rightarrow \operatorname{Hom}_{\Lambda}(C R, N)$ is the chain map that takes $\tilde{x}$ to $x$ and $\tilde{y}$ to $y$. These $t_{i}$ are the cochains we require. It is evident that

(i) $d t_{1}=t_{0}$,

(ii) $d t_{2 j}=\left(1-T^{-1}\right) t_{2 j-1}$,

(iii) $d t_{2 j+1}=N t_{2 j}$, and

(iv) $t_{p}=(-1)^{m(n+1)} m ! x^{p}$.

Now let $q=(n-2 k)(p-1)$, and for any integer $r$, define

$$
\nu(r)=(-1)^{m r(r-1) / 2}(m !)^{r} \quad[11, \text { p. 162]. }
$$

Finally, define

$$
\begin{aligned}
P^{k} x=(-1)^{k+m} \nu(-n-1)\left[\sum_{i=0}^{m}(-1)^{i} \psi^{*} D_{q+p-(2 i+1)}^{*} \chi\left(t_{2 i+1}\right)\right. & \\
& \left.-\sum_{i=1}^{m}(-1)^{i} \psi^{*} D_{q+p-2 i}^{*} \chi\left(\left(1-T^{-1}\right)^{p-2} t_{2 i}\right)\right]
\end{aligned}
$$

and

$$
\begin{aligned}
\beta P^{k} x=(-1)^{k+m} \nu(-n-1)\left[\sum_{i=0}^{m}(-1)^{i} \psi^{*} D_{q+p-1-(2 i+1)}^{*} \chi\left(t_{2 i+1}\right)\right. & \\
& \left.+\sum_{i=1}^{m}(-1)^{i} \psi^{*} D_{q+p-1-2 i}^{*} \chi\left(t_{2 i}\right)\right] .
\end{aligned}
$$

There are three important things to bear in mind about these imposing formulas. First, on the cochain level $P^{k}$ commutes and $\beta P^{k}$ anti-commutes with the boundary. 
Second, they define products and Steenrod operations on $H^{*} \operatorname{Hom}_{\Lambda}(C R, N)$ satisfying all the usual properties except that, in general, $P^{0} \neq 1[11, \mathrm{p} .198]$. Third, when applied to a cocycle, they reduce to the more familiar formulas used to define Steenrod operations (see for example [11, p. 182]).

The verification that $P^{k}$ commutes and $\beta P^{k}$ anti-commutes with the boundary is a straightforward computation. One need only note: (1) the properties of the $D_{k}$ as given in Lemma 3.1, (2) the formulas given for $d t_{i}$ in terms of $1-T^{-1}$ or $N$ applied to $t_{i-1}$, and (3) the fact that $N t_{p}=0$ and $(1-T)^{-1}=N$.

5. Bisimplicial Eilenberg-Zilber maps. For $\Lambda$ a Hopf algebra and $X(1), X(2), \ldots, X(p)$ bisimplicial $\Lambda$-modules, consider three different double complexes. First, consider the double complex which arises from the componentwise tensor product of the $X(i)$. Here we write $(X(1) \otimes X(2) \otimes \cdots \otimes X(p))_{s, t}=X(1)_{s, t} \otimes$ $X(2)_{s, t} \otimes \cdots \otimes X(p)_{s, t}$ for the resulting bisimplicial $\Lambda$-module. We use the tensor product face and degeneracy operators $d_{i}^{h}=d_{i}^{h} \otimes d_{i}^{h} \otimes \cdots \otimes d_{i}^{h}$, and $d_{i}^{v}=d_{i}^{v} \otimes d_{i}^{v}$ $\otimes \cdots \otimes d_{i}^{v}$, etc. The associated double complex is formed in the usual way, $d^{h}=\Sigma_{i}(-1)^{i} d_{i}^{h}, d^{v}=\Sigma_{j}(-1)^{j} d_{j}^{v}$ (i.e., first take the tensor product as bisimplicial modules and then form the double complex). Secondly, consider the bigraded tensor product of the double complexes $C X(i)$. This is written $C X(1) \hat{\otimes} C X(2)$ $\hat{\otimes} \ldots \hat{\otimes} C X(p)$ and has

$$
\begin{aligned}
(C X(1) \hat{\otimes} C X(2) \hat{\otimes} \cdots \hat{\otimes} C X(p))_{s, t} & \sum_{\substack{i_{1}+i_{2}+\cdots+i_{p}=s \\
j_{1}+j_{2}+\cdots+j_{p}=t}} C X(1)_{i_{1}, j_{1}} \otimes C X(2)_{i_{2}, j_{2}} \otimes \cdots \otimes C X(p)_{i_{p}, j_{p}} .
\end{aligned}
$$

It has vertical and horizontal differentials given by the Leibnitz rule (i.e., form the double complex $C X(i)$ for each $X(i)$ and then take the tensor product of these double complexes).

Thirdly, an intermediate object called the vertical tensor product of the $X(i)$ must be considered. This is the double complex $C\left(X(1) \otimes_{v} X(2) \otimes_{v} \cdots \otimes_{v} X(p)\right)$ given by

$$
\begin{aligned}
C\left(X(1) \otimes_{v} X(2) \otimes_{v} \cdots \otimes_{v}\right. & X(p))_{s, t} \\
& =\sum_{j_{1}+j_{2}+\cdots+j_{p}=t} X(1)_{s, j_{1}} \otimes_{v} X(2)_{s, j_{2}} \otimes_{v} \cdots \otimes_{v} X(p)_{s, j_{p}}
\end{aligned}
$$

with horizontal differential $d^{h}=\Sigma_{i}(-1)^{i} d_{i}^{h} \otimes d_{i}^{h} \otimes \cdots \otimes d_{i}^{h}$ and vertical differential given by the Leibnitz rule applied to the $d^{v}$ for each factor. Here we form a chain complex in the vertical direction for each $X(i)$ so that each $X(i)$ is in fact a simplicial chain complex. Next we take the dimensionwise tensor product of these chain complexes. Finally, we form a chain complex in the horizontal direction on the resulting simplicial chain complex.

Having established the preceding, we can begin to define bisimplicial EilenbergZilber maps. Fix a simplicial Eilenberg-Zilber map $\left\{D_{k}\right\}$ and define homomorphisms

$$
\tilde{G}_{q}: C(X(1) \otimes X(2) \otimes \cdots \otimes X(p)) \rightarrow C\left(X(1) \otimes_{v} X(2) \otimes_{v} \cdots \otimes_{v} X(p)\right)
$$


and

$H_{r}: C\left(X(1) \otimes_{v} X(2) \otimes_{v} \cdots \otimes_{v} X(p)\right) \rightarrow C X(1) \hat{\otimes} C X(2) \hat{\otimes} \cdots \hat{\otimes} C X(p)$, where $\tilde{G}_{q}$ is homogeneous of bidegree $(0, q)$ and $H_{r}$ is homogeneous of bidegree $(r, 0)$ as follows. The restriction of $\tilde{G}_{q}$ to $C(X(1) \otimes X(2) \otimes \cdots \otimes X(p))_{s, t}$ is

$$
\begin{aligned}
D_{q}\left(X(1)_{s, *}, X(2)_{s, *}, \ldots, X(p)_{s, *}\right): X(1)_{s, t} \otimes X(2)_{s, t} \otimes \cdots \otimes X(p)_{s, t} & \\
& \rightarrow \sum_{i_{1}+i_{2}+\cdots+i_{p}=t+q} X(1)_{s, i_{1}} \otimes X(2)_{s, i_{2}} \otimes \cdots \otimes X(p)_{s, i_{p}} .
\end{aligned}
$$

The restriction of $H_{r}$ to the summand

$$
X(1)_{s, i_{1}} \otimes X(2)_{s, i_{2}} \otimes \cdots \otimes X(p)_{s, i_{p}}\left(i_{1}+i_{2}+\cdots+i_{p}=t\right)
$$

is

$$
\begin{aligned}
D_{r}\left(X(1)_{*, i_{1}}, X(2)_{*, i_{2}}, \ldots,\right. & \left.X(p)_{*, i_{p}}\right): X(1)_{s, i_{1}} \otimes \cdots \otimes X(p)_{s, i_{p}} \\
& \rightarrow \sum_{j_{1}+j_{2}+\cdots+j_{p}=s+r} X(1)_{j_{1}, i_{1}} \otimes X(2)_{j_{2}, i_{2}} \otimes \cdots \otimes X(p)_{j_{p}, i_{p}} .
\end{aligned}
$$

Define the total differential $d$ on an element of bidegree $(s, t)$ to be $d^{h}+(-1)^{s} d^{v}$. Define $\varepsilon$ on an element of bidegree $(s, t)$ to be multiplication by $(-1)^{s t}$. Now let $G_{q}=\varepsilon \tilde{G}_{q} \varepsilon$. Then

(1) $d G_{q}+G_{q} d=G_{q-1}+T G_{q-1} T^{-1}$ ( $q$ odd),

(2) $d G_{q}-G_{q} d=G_{q-1}+T G_{q-1} T^{-1}+\cdots+T^{p-1} G_{q-1} T^{-p+1}$ ( $q$ even), and

(3) $d H_{r}+H_{r} d=H_{r-1}-T H_{r-1} T^{-1}$ ( $r$ odd),

(4) $d H_{r}-H_{r} d=H_{r-1}+T H_{r-1} T^{-1}+\cdots+T^{p-1} H_{r-1} T^{-p+1}$ ( $r$ even).

Next, for each integer $k \geqslant 0$, define a map

$$
\tilde{K}_{k}: C(X(1) \otimes X(2) \otimes \cdots \otimes X(p)) \rightarrow C X(1) \hat{\otimes} C X(2) \hat{\otimes} \cdots \hat{\otimes} C X(p)
$$

homogeneous of degree $k$ with respect to total degree by

$$
\tilde{K}_{k}=\sum_{\substack{r \text { even } \\ q \text { odd }}} T H_{r} T^{-1} G_{q}+\sum_{\substack{r \text { odd } \\ q \text { even }}} H_{r} G_{q} \quad(k \text { odd }),
$$

and

$$
\tilde{K}_{k}=\sum_{r, q \text { even }} H_{r} G_{q}+\sum_{0<n<m<p} \sum_{r, q \text { odd }}{ }_{r+q=k} T^{m} H_{r} T^{n-m} G_{q} T^{-n}
$$

for $k$ even. For $i_{1}, i_{2}, \ldots, i_{p}, j_{1}, j_{2}, \ldots, j_{p}$ integers, let

$$
\begin{aligned}
e\left(i_{1}, i_{2}, \ldots, i_{p}, j_{1}, j_{2}, \ldots, j_{p}\right)=i_{2}\left(j_{1}\right)+i_{3}\left(j_{1}+j_{2}\right)+\cdots & \\
& +i_{s}\left(j_{1}+j_{2}+\cdots+j_{s-1}\right)+\cdots+i_{p}\left(j_{1}+j_{2}+\cdots+j_{p-1}\right) .
\end{aligned}
$$

Now define $\sigma$ on an element $x_{1} \otimes x_{2} \otimes \cdots \otimes x_{p}$ in $X(1)_{i_{1}, j_{1}} \otimes X(2)_{i_{2}, j_{2}}$ $\otimes \cdots \otimes X(p)_{i_{p}, j_{p}}$ to be multiplication by

$$
(-1)^{e\left(i_{1}, i_{2}, \ldots, i_{p}, j_{1}, j_{2}, \ldots j_{p}\right)} \text {. }
$$

Finally, let $K_{k}=\sigma \tilde{K}_{k}$. 
A straightforward computation with the relations (1), (2), (3), and (4) proves the following.

LEMMA 5.1. For $\left\{K_{k}\right\}$ as defined above,

$$
\begin{aligned}
& d K_{k}+K_{k} d=K_{k-1}-T K_{k-1} T^{-1} \quad(k \text { odd }), \quad \text { and } \\
& d K_{k}-K_{k} d=K_{k-1}+T K_{k-1} T^{-1}+\cdots+T^{p-1} k_{k-1} T^{-p+1} \quad \text { (k even). }
\end{aligned}
$$

Thus, we call $\left\{K_{k}\right\}$ a bisimplicial Eilenberg-Zilber map.

Cohomology operations on $H^{*} \operatorname{Hom}_{\Lambda}(C X, N)$ can be now defined for any bisimplicial $\Lambda$-coalgebra $X$ and any commutative $\Lambda$-algebra $N$ by analogy with our definition for a simplicial $\Lambda$-coalgebra in $\$ 4$.

Each $K_{k}$ defines

$$
K_{k}^{*}: \operatorname{Hom}_{\Lambda}(C X(1) \hat{\otimes} \cdots \hat{\otimes} C X(p), N) \rightarrow \operatorname{Hom}_{\Lambda}(C(X(1) \otimes \cdots \otimes X(p)), N) \text {. }
$$

We also have

$$
\chi: \operatorname{Hom}_{\Lambda}(C X, N) \otimes \operatorname{Hom}_{\Lambda}(C X, N) \rightarrow \operatorname{Hom}_{\Lambda}(C X \hat{\otimes} C X, N)
$$

as well as

$$
\chi: \underbrace{\operatorname{Hom}_{\Lambda}(C X, N) \otimes \cdots \otimes \operatorname{Hom}_{\Lambda}(C X, N)}_{p} \rightarrow \operatorname{Hom}_{\Lambda} \underbrace{(C X \hat{\otimes} \cdots \hat{\otimes} C X, N)}_{p}
$$

defined as the similar maps for the simplicial case in $\$ 4$.

The cochain operations

$$
\begin{aligned}
& \mu: \operatorname{Hom}_{\Lambda}^{s}(C X, N) \otimes \operatorname{Hom}_{\Lambda}^{t}(C X, N) \rightarrow \operatorname{Hom}_{\Lambda}^{s+t}(C X, N), \\
& P^{k}: \operatorname{Hom}_{\Lambda}^{n}(C X, N) \rightarrow \operatorname{Hom}_{\Lambda}^{n+2 k(p-1)}(C X, N), \text { and } \\
& \beta P^{k}: \operatorname{Hom}_{\Lambda}^{n}(C X, N) \rightarrow \operatorname{Hom}_{\Lambda}^{n+2 k(p-1)+1}(C X, N)
\end{aligned}
$$

are defined exactly as before in $\$ 4$, on page 744 except that now we have a bisimplicial $\Lambda$-coalgebra and we use our bisimplicial Eilenberg-Zilber map $\left\{K_{k}\right\}$ in place of $\left\{D_{k}\right\}$. These operations pass to products and Steenrod operations on $H^{*} \operatorname{Hom}_{\Lambda}(C X, N)$.

The proof of Proposition 2.1 is exactly analogous to the proof of Proposition 1.1 in [16].

6. The spectral sequence of a bisimplicial $\Lambda$-coalgebra. If $X(1), X(2), \ldots, X(p)$ are bisimplicial $\Lambda$-coalgebras, $C X(1) \hat{\otimes} C X(2) \hat{\otimes} \ldots \hat{\otimes} C X(p)$ is filtered in the usual (increasing) way as a tensor product of filtered complexes:

$$
F_{k}(C X(1) \hat{\otimes} \cdots \hat{\otimes} C X(p))_{n}=\sum_{\substack{s+t=n \\ s \leqslant k}}(C X(1) \hat{\otimes} \cdots \hat{\otimes} C X(p))_{s, t} .
$$

We give $\operatorname{Hom}_{\Lambda}(C X(1) \hat{\otimes} \ldots \hat{\otimes} C X(p), N)$ the dual decreasing filtration:

$$
\begin{aligned}
F^{k} \operatorname{Hom}_{\Lambda}^{n}(C X(1) \hat{\otimes} \ldots \hat{\otimes} C X(p), N) \\
=\left\{f: C X(1) \hat{\otimes} \cdots \hat{\otimes} C X(p) \rightarrow N \mid f\left(F_{k-1}\right)=0\right\} .
\end{aligned}
$$


Then $\chi$ defined in $\S 5$ is filtration preserving, and if the simplicial Eilenberg-Zilber map $\left\{D_{k}\right\}$ used in defining $\left\{K_{k}\right\}$ is special, the following relations result:

$$
K_{k} F_{n} C(X(1) \otimes \cdots \otimes X(p)) \subset F_{n+k} C X(1) \hat{\otimes} \cdots \hat{\otimes} C X(p)
$$

and

$$
K_{k} F_{n} C(X(1) \otimes \cdots \otimes X(p)) \subset F_{n p} C X(1) \hat{\otimes} \cdots \hat{\otimes} C X(p) .
$$

These relations follow immediately from the definition of $K_{k}$ and the properties of a special Eilenberg-Zilber map. Dually we have

LEMMA 6.1. (a) $K_{k}^{*} F^{n} \subset F^{\operatorname{lig}(n / p)}$ and

LEMMA 6.1. (b) $K_{k}^{*} F^{n} \subset F^{n-k}$, where $\operatorname{lig}(x)$ denotes the least integer greater than or equal to $x$.

The spectral sequence $E_{r}=E_{r} \operatorname{Hom}_{\Lambda}(C X, N)$ is obtained in the usual way:

$$
E_{r}^{s, t}=Z_{r}^{s, t} /\left[\delta Z_{r-1}^{s-r+1, t+r-2}+Z_{r-1}^{s+1, t-1}\right]
$$

where

$$
Z_{r}^{s, t}=\left\{x \in F^{s} \operatorname{Hom}_{\Lambda}^{s+t}(C X, N) \mid \delta x \in F^{s+r} \operatorname{Hom}(C X, N)\right\} .
$$

The differential $\delta$ on $\operatorname{Hom}_{\Lambda}(C X, N)$ induces

$$
d_{r}: E_{r}^{s, t} \rightarrow E_{r}^{s+r, t-r+1} \text {. }
$$

Steenrod operations in this spectral sequence can now be defined. Recall that for $2 \leqslant r \leqslant n$ we let $B_{n}^{s, t}$ denote the subgroup of $E_{r}^{s, t}$ containing those elements which survive to $E_{n}^{s, t}$ and have zero residue there. We often write $B_{n}$ for $B_{n}^{s, t}$ when no confusion will ensue.

Proposition 6.2. Suppose that $r \geqslant 2$ and $x \in Z_{r}^{s, t}$.

Case 1. If $0 \leqslant 2 k \leqslant t$, then $P^{k} x \in Z_{r}^{s, t+2 k(p-1)}$, and $P^{k}$ passes to a homomorphism

$$
\rho^{k}: E_{r}^{s, t} \rightarrow E_{r}^{s, t+2 k(p-1)} \text {. }
$$

If $0 \leqslant 2 k<t$, then $\beta P^{k} x \in Z_{r}^{s, t+2 k(p-1)+1}$, and $\beta P^{k}$ passes to a homomorphism

$$
\beta^{\mathcal{N}}: E_{r}^{s, t} \rightarrow E_{r}^{s, t+2 k(p-1)+1} \text {. }
$$

Case 2. If $t \leqslant 2 k$, then $P^{k} x \in Z_{r}^{s+(2 k-t)(p-1), p t}$, and $\beta P^{k} \in Z_{r}^{s+(2 k-t)(p-1)+1, p t}$. For $t \leqslant 2 k<t+r-2$, these pass to homomorphisms in the spectral sequence:

$$
\begin{aligned}
\mathcal{P}^{k}: E_{r}^{s, t} & \rightarrow E_{r}^{s+(2 k-t)(p-1), p t} / B_{r-1+(2 k-t)(p-1)}, \\
\beta \mathcal{P}^{k}: E_{r}^{s, t} & \rightarrow E_{r}^{s+(2 k-t)(p-1)+1, p t} / B_{r+(2 k-t)(p-1)} .
\end{aligned}
$$

For $t+r-2 \leqslant 2 k$, these pass to homomorphisms

$$
\begin{aligned}
\mathcal{P}^{k}: E_{r}^{s, t} & \rightarrow E_{r}^{s+(2 k-t)(p-1), p t} / B_{p(r-2)+1}, \\
\beta \mathcal{P}^{k}: E_{r}^{s, t} & \rightarrow E_{r}^{s+(2 k-t)(p-1)+1, p t} / B_{p(r-2)+1} .
\end{aligned}
$$

PROOF. By the way of proof, we indicate here how to verify the location of the various elements in their proper filtrations and subgroups. Additivity of the operations will follow from their additivity at the $E_{2}$ stage to be provided by Proposition 7.1 . 
We begin by setting $n=s+t$, the total degree of $x \in Z_{r}^{s, t}$, and noting that $P^{k} x$ is given by a linear combination of terms

$$
\psi^{*} K_{(n-2 k)(p-1)}^{*} \chi(x \otimes x \otimes \cdots \otimes x)
$$

and

$$
\psi^{*} K_{(n-2 k)(p-1)+m}^{*} \chi(\alpha(m))
$$

for various values of $m$, where $\alpha(m)$ is a tensor product (in some order) of $m \delta x$ 's and $p-m x$ 's. The linear combination has been specifically chosen so that $\delta P^{k} x=P^{k} \delta x\left[11\right.$, p. 166]. Similar remarks hold for $\beta P^{k}$.

Case 1. First note that for $x \in Z_{r}^{s, t}$ (in particular $x \in F^{s} \operatorname{Hom}_{\Lambda}^{s+t}(C X, N)$ ), we have $\chi(x \otimes x \otimes \cdots \otimes x) \in F^{p s} \operatorname{Hom}_{\Lambda}(C X \hat{\otimes} \cdots \hat{\otimes} C X)$. Therefore, by (a) of Lemma 6.1, we have

$$
\psi^{*} K_{(n-2 k)(p-1)}^{*} \chi(x \otimes x \otimes \cdots \otimes x) \in F^{s} \operatorname{Hom}_{\Lambda}(C X, N) .
$$

Likewise, $x \in Z_{r}^{s, t}$ implies $\delta x \in F^{s+r}$ so that $\chi(\alpha(m)) \in F^{p s+m r}$, and thus $\psi^{*} K_{(n-2 k)(p-1)+m}^{*} \chi(\alpha(m)) \in F^{\operatorname{lig}((p s+m r) / p)} \subset F^{s}$. Thus $P^{k} x \in F^{s}$. Furthermore,

$$
\delta P^{k} x=P^{k} \delta x=(-1)^{k} \nu(-n-1) \psi^{*} K_{(n+1-2 k)(p-1)}^{*} \chi(\delta x \otimes \delta x \otimes \cdots \otimes \delta x)
$$

lies in $F^{s+r}$, verifying that $P^{k} x \in Z_{r}^{s, t+2 k(p-1)}$. Similar arguments show that if $x \in Z_{r-1}^{s+1, t-1}$, then $P^{k} x \in Z_{r-1}^{s+1, t+2 k(p-1)-1}$, and that if $x \in \delta Z_{r}^{s-r+1, t+r-2}$, then $P^{k} x \in \delta Z_{r}^{s-r+1, t+2 k(p-1)+r-2}$. The foregoing together with the additivity to be verified later show that $P^{k}$ passes to a homomorphism $\mathcal{P}^{k}: E_{r}^{s, t} \rightarrow E_{r}^{s, t+2 k(p-1)}$ for $0 \leqslant 2 k \leqslant t$. Identical arguments work for $\beta P^{k}$.

Case 2. This is similar to Case 1 except that here (b) of Lemma 6.1 is used. The indeterminacy in $\rho^{k}$ for $t \leqslant 2 k \leqslant t+r-2$, for example, can be seen as follows. If $x=\delta y$ with $y \in Z_{r-1}^{s-r+1, t+r-2}$ then $\delta P^{k} y=P^{k} x$, but $P^{k} y \in Z_{r-1}^{s-r+1, t+r-2+2 k(p-1)}$, so in the spectral sequence it is $d_{r-1+(2 k-t)(p-1)}$ that carries $P^{k} y$ to $P^{k} x$.

The proofs of Propositions 2.2 and 2.3 are immediate from the definitions. The proof of Proposition 2.4, as with that of Proposition 1.4 of [16], follows from the fact that $\rho=\bar{\rho} \lambda^{*}$ where $\bar{\rho}: F^{s} H^{s+t} \operatorname{Hom}_{\Lambda}(C X, N) \rightarrow E^{s, t}$ is the standard projection.

Proof of Proposition 2.5. Each of the cases of this proposition essentially follows from the relations $\delta P^{k} x=P^{k} \delta x$ and $\delta \beta P^{k} x=-\beta P^{k} \delta x$. The only thing to be verified is that the various elements involved survive long enough. Part of the proof of Case 2 illustrates how this is done. Suppose that $x \in Z_{r}^{s, t}$ represents $u \in E_{r}^{s, t}$. Then, repeating arguments in the proof of 6.2 , we find that $\delta x \in F^{s+r}$ and $P^{k} \delta x \in F^{s+r+(2 k-t+r-1)(p-1)}$. Since $\delta P^{k} x=P^{k} \delta x$, we have shown that $P^{k} x$ survives to $E_{r+(2 k-t+r-1)(p-1)}$. Furthermore, $\rho^{k} d_{r} u$ is represented by $P^{k} \delta x$, and this certainly survives to $E_{r+(2 k-t+r-1)(p-1)}$ because $\delta P^{k} \delta x=P^{k} \delta \delta x=0$. Case 4 also relies on the relations $\delta P^{k} x=P^{k} \delta x$ and $\delta \beta P^{k} x=-\beta P^{k} \delta x$. We simply note as in [11, p. 170] that, under the conditions of Case $4, \rho^{k} v$ and $\beta^{\rho^{k}} v$ are represented by $v u^{p-1}$ and $v^{p-1} u$ at $E_{r}$ and that these elements survive long enough. 
7. Operations on $E_{2}$. One representation of $E_{2}$ is that given in $\S 6$. Another expression is

$$
E_{2}^{s, t}=H_{h}^{s} H_{v}^{t} .
$$

$\operatorname{Hom}_{\Lambda}\left(C X_{s, t}, N\right)$ is a double complex and what is meant by the expression $H_{h}^{s} H_{v}^{t}$ is the $s$ th cohomology group of the complex

$$
\cdots \rightarrow H^{t} \operatorname{Hom}_{\Lambda}\left(C X_{s, *}, N\right) \stackrel{\delta^{h}}{\rightarrow} H^{t} \operatorname{Hom}_{\Lambda}\left(C X_{s+1, *}, N\right) \rightarrow \cdots
$$

Details on this representation can be found in [14,p. 68]. Using this representation, an element of $E_{2}$ can be represented by a $\Lambda$-homomorphism $x: X_{s, t} \rightarrow N$ satsifying $\delta^{v} x=0$ and $\delta^{h} x=\delta^{v} y$ for some $y: X_{s+1, t-1} \rightarrow N$.

Proposition 7.1. Suppose that $x: X_{s, t} \rightarrow N$ is a $\Lambda$-homomorphism representing $u \in E_{2}^{s, t}$.

Case 1. If $2 k \leqslant t$, then $\mathfrak{P}^{k} u \in E_{2}^{s, t+2 k(p-1)}$ is represented by the composition

$$
\begin{aligned}
X_{s, t+2 k(p-1)} & \stackrel{\psi}{\rightarrow} X_{s, t+2 k(p-1)} \otimes \cdots \otimes X_{s, t+2 k(p-1)} \\
(-1)^{k} \nu(-t) & \stackrel{D_{(t-2 k)(p-1)}}{\rightarrow} \sum_{j_{1}+j_{2}+\cdots+j_{p}=p t} X_{s, j_{1}} \otimes \cdots \otimes X_{s, j_{p}} \stackrel{\text { proj }}{\rightarrow} X_{s, t} \otimes \cdots \otimes X_{s, t} \\
x \otimes x \otimes \cdots \otimes x & \rightarrow{ }^{\rightarrow} N \otimes N \otimes \cdots \otimes N \stackrel{\mu}{\rightarrow} N .
\end{aligned}
$$

If $2 k<t$, then $\beta \mathscr{P}^{k} u \in E_{2}^{s, t+2 k(p-1)+1}$ is represented by the composition

$$
\begin{aligned}
X_{s, t+2 k(p-1)+1} & \stackrel{\psi}{\rightarrow} X_{s, t+2 k(p-1)+1} \otimes \cdots \otimes X_{s, t+2 k(p-1)+1} \\
(-1)^{k} \nu(-t) D_{(t-2 k)(p-1)-1} & \sum_{j_{1}+j_{2}+\cdots+j_{p}=p t} X_{s, j_{1}} \otimes \cdots \otimes X_{s, j_{p}} \stackrel{\text { proj }}{\rightarrow} X_{s, t} \otimes \cdots \otimes X_{s, t} \\
x \otimes x \otimes & \cdots \otimes x \\
& \rightarrow{ }^{\rightarrow} N \otimes N \otimes \ldots \otimes N \stackrel{\mu}{\rightarrow} N .
\end{aligned}
$$

Case 2. If $t \leqslant 2 k$, then $\mathcal{P}^{k} u \in E_{2}^{s+(2 k-1)(p-1), p t}$ is represented by the composition

$$
\begin{aligned}
& X_{s+(2 k-t)(p-1), p t} \stackrel{\psi}{\rightarrow} X_{s+(2 k-t)(p-1), p t} \otimes \cdots \otimes X_{s+(2 k-t)(p-1), p t} \\
& \stackrel{\nu(-t) D_{0}}{\rightarrow} \sum_{j_{1}+j_{2}+\cdots+j_{p}=p t} X_{s+(2 k-t)(p-1), j_{1}} \otimes \cdots \otimes X_{s+(2 k-t)(p-1), j_{p}} \\
& \stackrel{\text { proj }}{\rightarrow} X_{s+(2 k-t)(p-1), t} \otimes \cdots \otimes X_{s+(2 k-t)(p-1), t}
\end{aligned}
$$

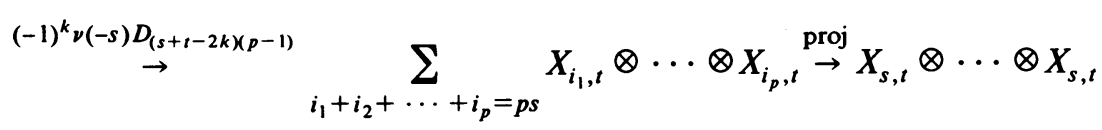

$$
\begin{aligned}
& \stackrel{x \otimes x}{\rightarrow} \underset{ }{\rightarrow} N \otimes N \otimes \cdots \otimes N \stackrel{\mu}{\rightarrow} N \text {, }
\end{aligned}
$$


and $\beta \mathscr{P}^{k} u \in E_{2}^{s+(2 k-t)(p-1), p t}$ is represented by the composition

$$
\begin{aligned}
& X_{s+(2 k-t)(p-1)+1, p t} \stackrel{\psi}{\rightarrow} X_{s+(2 k-t)(p-1)+1, p t} \otimes \cdots \otimes X_{s+(2 k-t)(p-1)+1, p t} \\
& \stackrel{\nu(-t) D_{0}}{\rightarrow} \sum_{j_{i}+j_{2}+\cdots+j_{p}=p t} X_{s+(2 k-t)(p-1)+1, j_{1}} \otimes \cdots \otimes X_{s+(2 k-t)(p-1)+1, j_{p}} \\
& \stackrel{\text { proj }}{\rightarrow} X_{s+(2 k-t)(p-1)+1, t} \otimes \cdots \otimes X_{s+(2 k-t)(p-1)+1, t}
\end{aligned}
$$

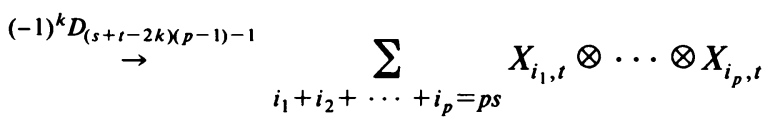

$$
\begin{aligned}
& \stackrel{\text { proj }}{\rightarrow} X_{s, t} \otimes \cdots \otimes X_{s, t} \stackrel{x \otimes x \otimes \cdots \otimes x}{\rightarrow} N \otimes N \otimes \cdots \otimes N \stackrel{\mu}{\rightarrow} N .
\end{aligned}
$$

Note there is no indeterminacy at the $E_{2}$ level.

Proof. We give the proof for $\mathscr{P}^{k} u$ in Case 1; the other cases are similar. In the original terminology $u$ is represented by a cochain of the form $x+y$ for some $y \in F^{s+1} \operatorname{Hom}_{\Lambda}(C X, N)$ and $\mathscr{P}^{k} u$ is represented by $P^{k}(x+y) \in$ $\Sigma_{i>s} \operatorname{Hom}_{\Lambda}\left(X_{i, j}, N\right)$. Thus we need only pick out the component of $P^{k}(x+y)$ which lies in $\operatorname{Hom}_{\Lambda}\left(X_{s, t+2 k(p-1)}, N\right)$. Using arguments like those in the proof of Proposition 6.2, we find that terms involving either $y$ or $\delta x$ are not in this component. We then need only identify the correct component of $\psi^{*} K_{(n-2 k)(p-1)}^{*} \chi(x \otimes x \otimes \cdots \otimes x)$. The definition of $K^{*}$ and the observation that the composition

$$
\begin{gathered}
X_{s, t} \otimes \cdots \otimes X_{s, t} \stackrel{\substack{D_{s(p-1)}}}{\rightarrow} \sum_{i_{1}+i_{2}+\cdots+i_{p}=p s} X_{i_{1}, t} \otimes \cdots \otimes X_{i_{p}, t} \\
\stackrel{\text { proj }}{\rightarrow} X_{s, t} \otimes \cdots \otimes X_{s, t}
\end{gathered}
$$

is multiplicaion by $(m !)^{s}(-1)^{m\left(s^{2}+s\right) / 2}$ (remember that $\left\{D_{k}\right\}$ is a special EilenbergZilber map) verifies that we have in fact selected the proper composition.

The astute reader will have noticed that, if $x \in E_{r}^{s, t}$ and $t$ is odd, the horizontal Steenrod operations (cf. $\$ 2$, p. 740) seem to be shifted to the left by $p-1$ from their expected location. That is, an ordinary Steenrod power operation increases dimension by even multiples of $p-1$, but for $t$ odd, our horizontal operations increase dimension by odd multiples of $p-1$. (Of course, total degree is always raised by even multiples of $p-1$.) The apparent shift in the horizontal operations happens because our horizontal operations at the $E_{2}$ level are defined in the cohomology of a graded commutative coalgebra. Let $\pi$ be the cyclic group of order $p$ contained in $\Sigma_{p}$, the symmetric group on $p$ letters. Let $Z_{p}^{ \pm}$be the integers $\bmod p$ as a $\pi$-module with $\pi$-action given by the sign of the permutation. May [11,p. 159] shows that the induced map

$$
H_{i}\left(\pi, Z_{p}^{ \pm}\right) \rightarrow H_{i}\left(\Sigma_{p}, Z_{p}^{ \pm}\right)
$$

is zero except when $i=(2 j+1)(p-1)$ or $i=(2 j+1)(p-1)-1$. Thus our horizontal operations are only nonzero ones possible. 


\section{REFERENCES}

1. S. Araki, Steenrod reduced powers in the spectral sequences associated with a fibering. I, II, Mem. Fac. Sci. Kyushu Univ. Ser. A Math. 11 (1957), 15-64, 81-97.

2. A. Dold, Über die Steenrodschen Kohomologieoperationen, Ann. of Math. (2) 73 (1961), 258-294.

3. W. G. Dwyer, Homotopy operations for simplicial commutative algebras, Trans. Amer. Math. Soc. 260 (1980), 421-435.

4. Higher divided squares in second-quadrant spectral sequences, Trans. Amer. Math. Soc. 260 (1980), 437-447.

5. L. Kristensen, On the cohomology of two-stage Postnikov systems, Acta Math. 107 (1962), 73-123.

6. T. Kudo, A transgression theorem, Mem. Fac. Sci. Kyushu Univ. Ser. A Math. 9 (1956), 79-81.

7. T.-C. Kuo, A family of spectral operations, Proc. Nat. Acad. Sci. U. S. A. 53 (1965), 658-661.

8. S. Mac Lane, Homology, Springer-Verlag, New York, 1967.

9. W. S. Massey, Some problems in algebraic topology and theory of fibre bundles, Ann. of Math. (2) 62 (1955), 327-359.

10. J. P. May, Simplicial objects in algebraic topology, Van Nostrand, New York, 1967.

11. , A general approach to Steenrod operations, The Steenrod Algebra and its Applications, Lecture Notes in Math., vol. 168, Springer-Verlag, New York, 1970, pp. 153-231.

12. M. Mori, A note on Steenrod operations in the Eilenberg-Moore spectral sequence, Proc. Japan Acad. Ser. A Math. Sci. 53 (1977), 112-114. , The Steenrod operations in the Eilenberg-Moore spectral sequence, Hiroshima Math. J. 9 (1979), 17-34.

14. R. E. Mosher and M. C. Tangora, Cohomology operations and applications in homotopy theory, Harper and Row, New York, 1968.

15. D. Rector, Steenrod operations in the Eilenberg-Moore spectral sequence, Comment. Math. Helv. 45 (1970), 540-552.

16. W. M. Singer, Steenrod squares in spectral sequences. I, Trans. Amer. Math. Soc. 175 (1973), $327-336$.

17. __ Steenrod squares in spectral sequences. II, Trans. Amer. Math. Soc. 175 (1973), 337-353.

18. L. Smith, On the Kunneth theorem. I, Math. Z. 116 (1970), 94-140.

19. E. H. Spanier, Algebraic topology, McGraw-Hill, New York, 1966.

20. N. E. Steenrod and D. B. A. Epstein, Cohomology operations, Princeton University Press, Princeton, N. J., 1962.

21. R. M. Switzer, Algebraic topology-Homotopy and homology, Springer-Verlag, New York, 1975.

22. R. Vazquez, Note on Steenrod squares in the spectral sequence of a fiber space, Bol. Soc. Mat. Mexicana (2) 2 (1957), 1-8.

Department of Mathematics, University of Santa Clara, Santa Clara, California 95053 